\title{
Interaction and Surface Modification of Biomaterials with Cells Based on Carbon Nanomaterials
}

\author{
Vajradhar Lather \\ ${ }^{1}$ Department of Chemistry, Karnatak University, Dharwad, Karnataka, 580003, India
}

\begin{abstract}
Keywords: Carbon nanomaterials; biological materials; cell interactions; surface decoration
Abstract. Carbon-based nanomaterials, such as carbon nanomaterials, play an important role in many promising nanomaterials in the field of biomedicine. Among them, carbon nanomaterials are a new kind of porous carbon nanomaterials with great application potential. Therefore, based on carbon nanomaterials, the interaction between biomaterials and cells and surface modification were analyzed. Studies have shown that the interaction between the surface of biological materials and cells is mainly the mutual molecular recognition between the surface receptors of cell membranes and the ligands on the surface of biomaterials. Therefore, biomimetic modification of the surface of biomaterials is used to enhance cell affinity and specific recognition. The carbon nanomaterial has a large specific surface area and pore volume, can provide high drug-loading capacity, has an adjustable pore structure and pores, can control the release of the drug molecules, and has a good application prospect in the field of drug delivery systems.
\end{abstract}

\section{Introduction}

Carbon nanomaterials mainly include fullerenes, carbon nanomaterials and graphene, which are indispensable materials in nanoscience and technology [1]. This is another major discovery in the field of carbon chemistry since the discovery of C60 by foreign scholars in 1985. It has aroused people's extensive interest and has become a major research hotspot in the field of fullerenes. It is the most advanced in the fields of physics, chemistry and materials science. One of the research areas [2]. Through acidification treatment, ligand binding and other experimental methods, the polarity, water dispersibility, surface charge, ion release, etc. of nanomaterials can be effectively changed, so that nanomaterials have different biological effects [3]. Its basic strategy is to simulate physiological environment in vitro, adhere tissue cells to porous scaffolds with good biocompatibility and biodegradable absorption, and provide nutrition to expand them to form a cell-scaffold complex, then implant the complex into the body [4]. Because nano-materials often have large specific surface area and strong adsorption capacity, and have unique surface characteristics. At the same time, nano-scale materials can also be swallowed by cells through interstitial space, and can also pass through the smallest capillaries of the human body and even through the blood-brain barrier [5]. Therefore, it has broad application prospects as a drug carrier in the field of biomedicine. Among this, the use of carbon nanomaterials in immunotherapy has drawn a lot of attention for the treatment of several different types of cancer, including glioblastoma [6], pancreatic cancer [7], and leukemia [8]. In addition, carbon nanomaterials also have high toughness and strength, and have a large specific surface area [4]. These unique characteristics make carbon nanomaterials become a research hotspot in recent years.

Nanomaterials refer to materials with at least one dimension less than $100 \mathrm{~nm}$ or consisting of them as basic units in three-dimensional space [9]. Because the axial resistance of carbon nanomaterials is very small, they can be transformed into superconductors at low temperature. One can be regarded as one-dimensional quantum material [2]. The unique physical and chemical properties of nanomaterials, such as size, shape, composition, surface functionalization and surface charge, will affect their distribution and interaction with tissues and cells in vivo. Therefore, in the medical application of nanomaterials, biological safety is one of the focuses of attention of scientists and technicians [10]. All new nanomaterials must be carefully and rigorously evaluated before they are applied to the clinic to fully understand their physicochemical properties and possible adverse reactions, and to clarify their biosafety. Nano-materials with different degrees of carboxylation modification can be obtained by using 60Co radiation multi-wall carbon nanomaterials and 
acidification treatment [10]. Scientists predict that CNTs will be the most promising one-dimensional nanomaterials, nanoelectronic device materials and new generation flat panel display materials in the 21 st century. Because of its excellent electrical, thermal and mechanical properties and large specific surface area, graphene has a wide range of applications in hydrogen storage materials, supercapacitors, high-efficiency catalysts and nano-biosensing.

\section{Carbon nanomaterial}

\section{Structure of carbon nanomaterials}

Carbon nanomaterials are an important member of the fullerene family and are another allotrope of crystalline carbon. From graphite, diamond to fullerenes, to carbon nanomaterials, the structure of crystalline carbon is increasingly perfect (from zero-dimensional to three-dimensional). However, two problems have always hindered the entry of carbon nanomaterials into applications: (1) Carbon nanomaterials produced by various methods are mixtures of metallic and semiconducting carbon nanomaterials. (2) Due to the van der Waals force, carbon nanomaterials are easily agglomerated, resulting in difficulty in dispersing in a solvent, and are difficult to dissolve and operate in almost all solvents, which greatly limits their application. Unlike Ci GNP and PVP GNP, CTAB GNP can destroy keratinocytes to connect closely with gold nanoparticles. Cells can respond to micro- and nano-scale surface topography and generate contact guidance when recognizing material surface characteristics. So people can use material surface topography to regulate cell behavior. For highly dispersed drug molecules, carbon has large specific surface area and pore volume, which can provide high drug loading capacity. It has adjustable ordered structure and pore, and can control the release of drug molecules. Therefore, it has great application prospects as a drug delivery system. The cross section of the tube is mostly graphite hexagonal structure, and the top of the tube is mostly composed of pentagonal or heptagonal carbon rings.

\section{Properties of Carbon Nanomaterials}

Carbon nanomaterials are mainly composed of carbon elements. The surface modification of carbon nanomaterials can be divided into covalent bond and non-covalent bond modification. Lowdose gold nanorods can enter red blood cells and interact with the highest abundant hemoglobin in the cells, forming gold nanorods-protein complexes by electrostatic force, which can cause significant changes in the spatial structure of hemoglobin and hinder the release of oxygen by proteins. Microstructure domains on the surface of biomaterials can regulate signal transduction between cells and matrix, thus affecting the formation of cell adhesion domains and the development of cytoskeleton, and ultimately forming highly oriented cell patterns. Carbon nanomaterials have various shapes, such as cylindrical, coil, ring and bamboo. CNDs not only have excellent fluorescence characteristics but also no cytotoxicity, so they have good application prospects in labeling biomolecules for fluorescence imaging. There are a variety of methods for dispersing carbon nanomaterials, and the surface of the carbon nanomaterials is modified to achieve separation and dissolution. The main methods are covalent bond modification and non-covalent bond modification. The carbon nanomaterial is a seamless nanotubular structure formed by curling a single layer or a plurality of graphite sheets, and the spacing between adjacent layers is equivalent to the layer spacing of graphite, which is about $0.34 \mathrm{~nm}$. Carbon nanomaterials have a diameter of a few nanometers to tens of nanometers, a length of tens of nanometers to micrometers, and an ultra-long carbon nanomaterial with a length of $2 \mathrm{~mm}$.

The effects of MCN, MCN-PVP and MCN-PEG on L929 and HeLa cells after 24 hours of inoculation on the active oxygen of the cells are shown in Fig. 1 and Fig. 2. For both cells, modified mesoporous carbon nanoparticles (MCN-PVP and MCN-PEG-masters can significantly reduce ROS production compared to pure mesoporous carbon nanoparticles. Decrease mesoporous carbon nanoparticles to cells The level of oxidative stress increases the biocompatibility of the carrier material. 


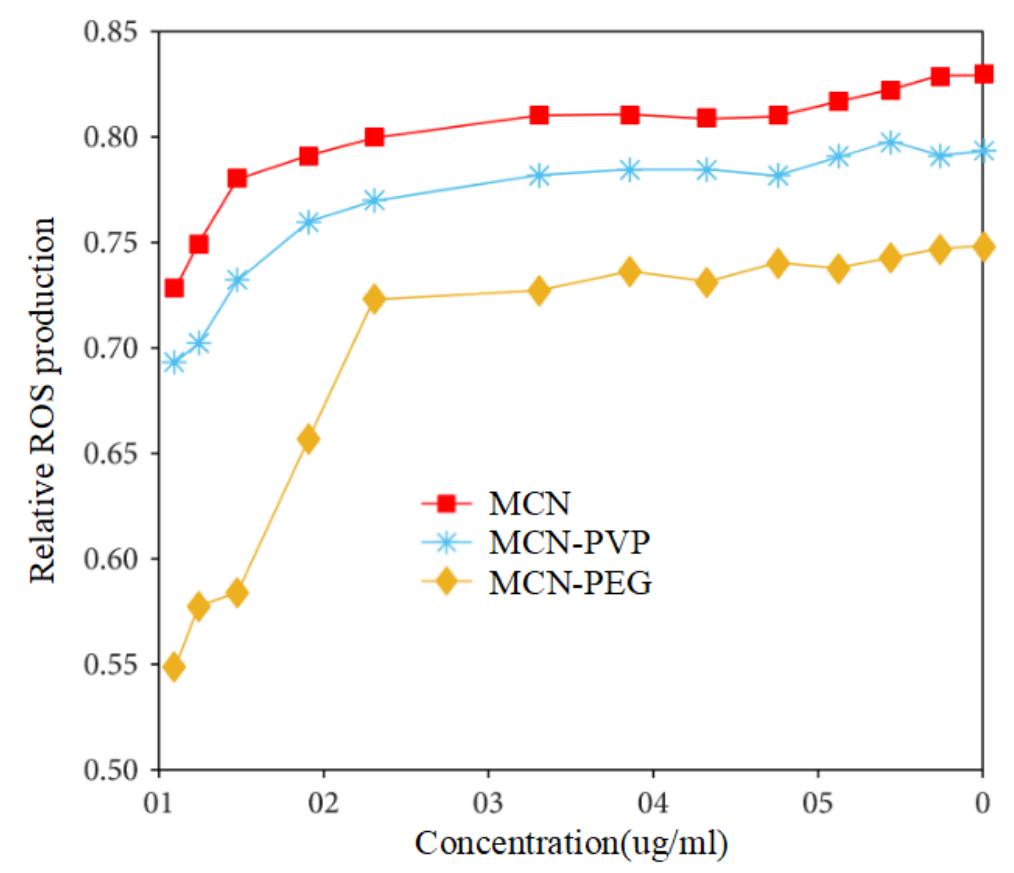

Fig.1 Effect of carbon nanomaterials on reactive oxygen species in L929 cells

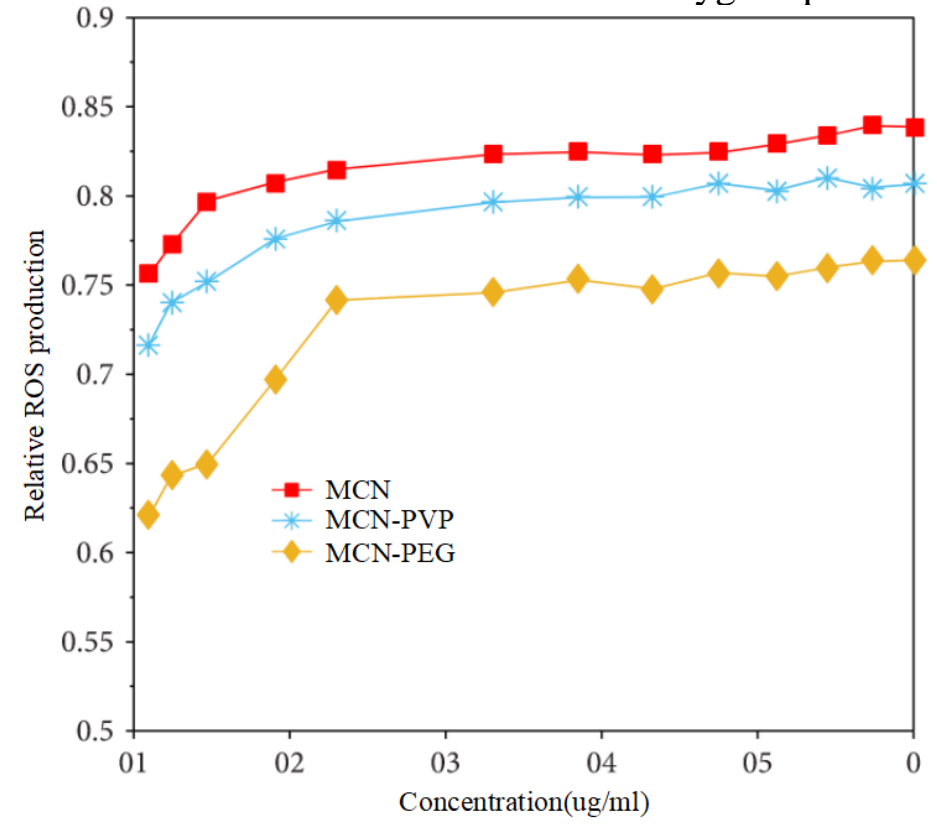

Fig.2 Effect of Carbon Nanomaterials on Reactive Oxygen Species in HeLa Cells

\section{Biomimetic Study on Surface Properties of Biomaterials}

\section{Topological Structure of Biomaterial Surface}

From the point of view of material science, the material structure determines the material properties, so the surface microstructures have a significant impact on the surface properties. Because of the simplicity, rapidity and sensitivity of electrochemical methods, and the maturity of electrode miniaturization and surface modification technology, the electrode not only greatly improves the sensitivity, but also has high recognition specificity through Ingenious modification and molecular self-assembly. The yellowish methylreductant dye produced by the redox of water-soluble tetradecyl salts by intracellular deaminase can be dissolved in tissue culture medium, and the amount of methylreductant produced is proportional to the number of living cells. He filled the anode with powders of memory and nickel metal, and obtained single-walled carbon nano-materials of gram stars by stone-lift arc discharge method. Due to its large specific surface area and planar conjugate structure, GO can interact with biomolecules such as DNA and proteins for bioanalysis and 
bioluminescence imaging. Covalent bond modification destroys the aromatic structure of some carbon atoms on carbon nanomaterials, resulting in a decrease in the electrical properties of carbon nanomaterials. Non-covalent bond modification can avoid this, thus maintaining the perfect structure of surface carbon atoms. application. Therefore, the use of electrochemical microsensors will make the dream of real-time detection and monitoring of intracellular signal molecules a reality.

\section{Hydrophilic/hydrophobic balance on the surface of biomaterials}

Generally speaking, the hydrophilic surface promotes cell adhesion, and the hydrophobic surface has a strong adsorption function for proteins. Therefore, the research on carbon nanomaterials mainly focuses on the above two aspects. Studies have shown that many types of compounds can react with carbon nanomaterials to form soluble supramolecular complexes that separate the diameter and chirality of carbon nanomaterials. In most cases, bacterial cells do not secrete redox active mediators or electron transport proteins, and electron transport can only be promoted when bacterial cells are in direct contact with the electrodes, thereby producing changes in electrode charge and potential and generating electrical current. This is related to the negative binding of nanosilver to the KKS-initiating zymogen FXII and its autoactivation. The activation of KKS can regulate the internalization and degradation of vascular endothelial cell adhesion junction protein, resulting in increased vascular permeability. The adhesion between cells and materials is mediated by proteins. Excessive hydrophilic surface is not conducive to the adsorption of proteins. Therefore, there is an optimal hydrophilic/hydrophobic balance on the surface suitable for cell adhesion and growth, which varies with different types of cells. Compared with unmodified carbon nanoparticles, modified carbon nanoparticles (MCN-PVP and MCN-PEG) can significantly reduce ROS production and reduce the role of carbon nanoparticles in stimulating oxidative stress. However, the main disadvantage of this method is that the obtained carbon nanomaterials have uncertain spatial orientation, easy sintering and high impurity content.

\section{Conclusion}

In summary, carbon nanomaterials have been a hot topic in the research of nanoscience and technology, and important research progress has been made due to their unique structure and excellent physical and chemical properties. It has attracted much attention in lithium-ion battery materials, photoelectric materials, catalyst carriers, chemical and biological sensors, hydrogen storage materials and supercapacitor materials. The construction of cell compatibility interface plays a key role in promoting the development of cell sensors, tissue engineering and clinical application of tissue engineering materials. Surface modification aims to mediate the interaction between material surface and cells, regulate the two-way dynamic balance of cell growth and apoptosis on material surface, and create a good artificial ECMs environment for cells. Cell apoptosis assay results also showed that modified and unmodified carbon nanoparticles did not induce apoptosis in L929 and HeLa cells. In order to effectively synthesize carbon nanometers, it is also possible to use a plasma-enhanced or microwave-assisted method to maintain a uniform distribution of carbon source. The oxidation product of DAB quenches its fluorescence by causing aggregation of carbon dots, and based on this, a fluorescence quenching method is established to achieve highly sensitive detection of hydrogen peroxide and glucose. How to achieve high-quality preparation of high-quality carbon nanomaterials and their supramolecular functional assembly is a problem that must be solved in the process of carbon nanomaterials from research to application.

\section{References}

1 Y. Deng, Y. S. Ok, D. Mohan, C. U. Pittman Jr, and X. Dou, Carbamazepine removal from water by carbon dot-modified magnetic carbon nanotubes. Environmental research. 169, 434-444 (2019)

2 A. Omrani, E. Esmaeilzadeh, M. Jafari, and A. Behzadmehr, Effects of multi walled carbon nanotubes shape and size on thermal conductivity and viscosity of nanofluids. Diamond and Related Materials. 93, 96-104 (2019) 
3 S. Fiorito, Carbon nanotubes: angels or demons?, CRC Press, (2019)

4 J. Yoon, and C. Ru, Metamaterial-like vibration of doublewalled carbon nanotubes. Physica E: Low-dimensional Systems and Nanostructures. 107, 196-202 (2019)

5 R. Abbaslou, and A. Dalai, Google Patents, (2019)

6 M. Zheng, in Single-Walled Carbon Nanotubes, Springer, (2019), pp. 129-164.

7 K. B. Knudsen, T. Berthing, P. Jackson, S. S. Poulsen, A. Mortensen, N. R. Jacobsen, V. Skaug, J. Szarek, K. S. Hougaard, and H. Wolff, Physicochemical predictors of Multi-Walled Carbon Nanotube-induced pulmonary histopathology and toxicity one year after pulmonary deposition of 11 different Multi-Walled Carbon Nanotubes in mice. Basic \& clinical pharmacology \& toxicology. 124, 211-227 (2019)

8 E. Fleming, F. Du, E. Ou, L. Dai, and L. Shi, Thermal conductivity of carbon nanotubes grown by catalyst-free chemical vapor deposition in nanopores. Carbon. 145, 195-200 (2019)

9 R. Zhang, and B. D. Ulery, Synthetic vaccine characterization and design. Journal of Bionanoscience. 12, 1-11 (2018)

10 A. A. Chaudhari, S. Joshi, K. Vig, R. Sahu, S. Dixit, R. Baganizi, V. A. Dennis, S. R. Singh, and S. Pillai, A three-dimensional human skin model to evaluate the inhibition of Staphylococcus aureus by antimicrobial peptide-functionalized silver carbon nanotubes. Journal of biomaterials applications. 33, 924-934 (2019)

11 R. Fang, K. Chen, L. Yin, Z. Sun, F. Li, and H. M. Cheng, The Regulating Role of Carbon Nanotubes and Graphene in Lithium-Ion and Lithium-Sulfur Batteries. Advanced Materials. 31, 1800863 (2019)

12 R. Zhang, M. M. Billingsley, and M. J. Mitchell, Biomaterials for vaccine-based cancer immunotherapy. Journal of Controlled Release. (2018)

13 R. Zhang, J. S. Kramer, J. D. Smith, B. N. Allen, C. N. Leeper, X. Li, L. D. Morton, F. Gallazzi, and B. D. Ulery, Vaccine Adjuvant Incorporation Strategy Dictates Peptide Amphiphile Micelle Immunostimulatory Capacity. The AAPS journal. 20, 73 (2018)

14 K. A. Whitehead, R. Langer, and D. G. Anderson, Knocking down barriers: advances in siRNA delivery. Nature reviews Drug discovery. 8, 129 (2009)

15 D. R. Wilson, R. Sen, J. C. Sunshine, D. M. Pardoll, J. J. Green, and Y. J. Kim, Biodegradable STING agonist nanoparticles for enhanced cancer immunotherapy. Nanomedicine: Nanotechnology, Biology and Medicine. 14, 237-246 (2018)

16 Q. Wu, M. Chen, M. Buchwald, and R. A. Phillips, A simple, rapid method for isolation of high quality genomic DNA from animal tissues. Nucleic acids research. 23, 5087 (1995)

17 R. Xavier, and D. Podolsky, Unravelling the pathogenesis of inflammatory bowel disease. Nature. 448, 427 (2007)

18 Z. Yaari, D. Da Silva, A. Zinger, E. Goldman, A. Kajal, R. Tshuva, E. Barak, N. Dahan, D. Hershkovitz, and M. Goldfeder, Theranostic barcoded nanoparticles for personalized cancer medicine. Nature communications. 7, 13325 (2016)

19 A. J. Mukalel, R. S. Riley, R. Zhang, and M. J. Mitchell, Nanoparticles for Nucleic Acid Delivery: Applications in Cancer Immunotherapy. Cancer letters. (2019)

20 J. D. Smith, L. N. Cardwell, D. Porciani, J. A. Nguyen, R. Zhang, F. Gallazzi, R. R. Tata, D. H. Burke, M. A. Daniels, and B. D. Ulery, Aptamer-displaying peptide amphiphile micelles as a celltargeted delivery vehicle of peptide cargoes. Physical biology. 15, 065006 (2018) 
21 T. Ouyang, Y. Q. Ye, C. Y. Wu, K. Xiao, and Z. Q. Liu, Heterostructures Composed of N-Doped Carbon Nanotubes Encapsulating Cobalt and $\beta$-Mo2C Nanoparticles as Bifunctional Electrodes for Water Splitting. Angewandte Chemie International Edition. 58, 4923-4928 (2019)

22 Z. Noorimotlagh, S. A. Mirzaee, S. S. Martinez, S. Alavi, M. Ahmadi, and N. Jaafarzadeh, Adsorption of textile dye in activated carbons prepared from DVD and CD wastes modified with multi-wall carbon nanotubes: Equilibrium isotherms, kinetics and thermodynamic study. Chemical Engineering Research and Design. 141, 290-301 (2019)

23 X. Jia, and F. Wei, in Single-Walled Carbon Nanotubes, Springer, (2019), pp. 299-333.

24 R. Zhang, J. D. Smith, B. N. Allen, J. S. Kramer, M. Schauflinger, and B. D. Ulery, Peptide Amphiphile Micelle Vaccine Size and Charge Influence the Host Antibody Response. ACS Biomaterials Science \& Engineering. 4, 2463-2472 (2018)

25 R. Akbarzadeh, M. Ghaedi, S. N. Kokhdan, and D. Vashaee, Remarkably improved electrochemical hydrogen storage by multi-walled carbon nanotubes decorated with nanoporous bimetallic Fe-Ag/TiO 2 nanoparticles. Dalton Transactions. 48, 898-907 (2019)

26 M. L. Yola, and N. Atar, Simultaneous determination of $\beta$-agonists on hexagonal boron nitride nanosheets/multi-walled carbon nanotubes nanocomposite modified glassy carbon electrode. Materials Science and Engineering: C. 96, 669-676 (2019)

27 R. Zhang, C. N. Leeper, X. Wang, T. A. White, and B. D. Ulery, Immunomodulatory vasoactive intestinal peptide amphiphile micelles. Biomaterials science. 6, 1717-1722 (2018)

28 X. Shen, and D. R. Corey, Chemistry, mechanism and clinical status of antisense oligonucleotides and duplex RNAs. Nucleic acids research. 46, 1584-1600 (2017)

29 J. Viger-Gravel, A. Schantz, A. C. Pinon, A. J. Rossini, S. Schantz, and L. Emsley, Structure of Lipid Nanoparticles Containing siRNA or mRNA by Dynamic Nuclear Polarization-Enhanced NMR Spectroscopy. The Journal of Physical Chemistry B. 122, 2073-2081 (2018)

30 L. Warren, P. D. Manos, T. Ahfeldt, Y.-H. Loh, H. Li, F. Lau, W. Ebina, P. K. Mandal, Z. D. Smith, and A. Meissner, Highly efficient reprogramming to pluripotency and directed differentiation of human cells with synthetic modified mRNA. Cell stem cell. 7, 618-630 (2010)

31 R. Zhang, L. D. Morton, J. D. Smith, F. Gallazzi, T. A. White, and B. D. Ulery, Instructive Design of Triblock Peptide Amphiphiles for Structurally Complex Micelle Fabrication. ACS Biomaterials Science \& Engineering. (2018)

32 C. J. Barnett, C. Evans, J. E. McCormack, C. E. Gowenlock, P. Dunstan, W. Adams, A. OrbaekWhite, and A. R. Barron, Experimental measurement of angular and overlap dependence of conduction between carbon nanotubes of identical chirality and diameter. Nano Letters. (2019)

33 I. Jeon, Y. Matsuo, and S. Maruyama, in Single-Walled Carbon Nanotubes, Springer, (2019), pp. 271-298.

34 P. Mu, Z. Zhang, W. Bai, J. He, H. Sun, Z. Zhu, W. Liang, and A. Li, Superwetting Monolithic Hollow-Carbon-Nanotubes Aerogels with Hierarchically Nanoporous Structure for Efficient Solar Steam Generation. Advanced Energy Materials. 9, 1802158 (2019)

35 P. G. Guimaraes, R. Zhang, R. Spektor, M. Tan, A. Chung, M. M. Billingsley, R. El-Mayta, R. S. Riley, L. Wang, J. M. Wilson, and M. J. Mitchell. Ionizable lipid nanoparticles encapsulating barcoded mRNA for accelerated in vivo delivery screening” Journal of Controlled Release (2019)

36 M. Billingsley, N. Singh, P. Ravikumar, R. Zhang, C. H. June, M. J. Mitchell. "Ionizable lipid nanoparticle mediated mRNA delivery for human CAR T cell engineering" Nano Letters (2020). Mar 11;20(3):1578-1589 
37 A. Marabelle, H. Kohrt, C. Caux, R. Levy, Intratumoral immunization: a new paradigm for cancer therapy, Clinical Cancer Research, 20 (2014) 1747-1756.

38 C. Leonhardt, G. Schwake, T.R. Stögbauer, S. Rappl, J.-T. Kuhr, T.S. Ligon, J.O. Rädler, Singlecell mRNA transfection studies: delivery, kinetics and statistics by numbers, Nanomedicine: Nanotechnology, Biology and Medicine, 10 (2014) 679-688. 